\title{
Neonatal Abstinence Syndrome: Influence of a Combined Inpatient/Outpatient Methadone Treatment Regimen on the Average Length of Stay of a Medicaid NICU Population
}

\author{
Jerry Lee, MD,' Sonia Hulman, MD, MBA, Michael Musci Jr., DO, MBA,, and Ellen Stang, MD¹
}

\begin{abstract}
Prescription opioid and heroin abuse have been increasing steadily year after year, and continue to be a serious national problem. A sequela of the increase in opioid abuse has been an increase in the number of infants born with opioid dependence. These infants often require costly, prolonged stays in the neonatal intensive care unit (NICU) for drug withdrawal treatment. The authors studied a population of infants from a large Medicaid health plan who were born with neonatal abstinence syndrome (NAS) secondary to in utero opioid exposure to assess the average length of stay in the NICU, and to determine the variables that may account for differences in interinstitutional lengths of stay. The overall average length of stay for NAS was 21.1 days for the 139 infants included in the study. Analysis of the medication used for treatment revealed that infants who were treated with a combined inpatient/outpatient regimen with methadone had an average length of stay of 11.4 days versus 25.1 days for infants who were treated entirely as inpatients $(P<0.001)$, a $55 \%$ reduction in average length of stay. In 2009 there were an estimated 13,600 cases of NAS in the United States at a cost of $\$ 53,000$ per case. A 55\% reduction in length of stay corresponds to \$396 million in annual savings for the treatment of NAS. Development of successful combined inpatient/outpatient management programs for NAS warrants further consideration. (Population Health Management 2015;18:392-397)
\end{abstract}

\section{Introduction}

$\mathbf{P}$ RESCRIPTION DRUG ABUSE in the United States has reached epidemic proportions. Comprising $4.6 \%$ of the world's population, Americans consume $80 \%$ of the global opioid supply, $99 \%$ of the global hydrocodone supply, and two thirds of all illegal drugs. ${ }^{1}$ Hydrocodone remains the most commonly prescribed drug in the United States, with more than 131 million prescriptions issued in $2010 .^{2}$ Prescription opioid abuse has increased by $41 \%$ between 1998 and 2008. ${ }^{3}$

It has been estimated that illicit drug use occurs in $4.4 \%$ of pregnant women, $16.2 \%$ among pregnant teens, and $7.4 \%$ among pregnant women aged 18 to 25 years. ${ }^{4}$ As can be expected with the increasing use of prescription drugs, the incidence of neonatal abstinence syndrome (NAS) will increase and remain an ongoing problem in the neonatal population. ${ }^{5}$ Between 2000 and 2009, the number of pregnant women using opioids during pregnancy has increased from 1.2 to 5.6 per 1000 live births, and the rate of NAS in the United States has increased from 1.2 to 3.4 per 1000 live births, resulting in an estimated 13,600 cases of NAS in $2009 .^{6}$

NAS was first described in the 1960s as a constellation of symptoms seen in neonates born to mothers who were using illicit opioids or methadone during their pregnancy. ${ }^{5}$ Opioids are a class of compounds that activate opioid receptors in the central nervous system to produce supraspinal analgesia. Other effects include sedation, euphoria, decreased gastrointestinal motility, and respiratory depression. In the neonate, the predominant signs and symptoms of acute opioid withdrawal involve central nervous system irritability, gastrointestinal tract dysfunction, and autonomic overactivity. This syndrome can present as tremors, irritability, high-pitched crying, increased muscle tone, frequent yawning and sneezing, seizures, poor feeding, vomiting, diarrhea, dehydration, failure to thrive, sweating, nasal congestion, and temperature instability. This constellation of findings has been termed the neonatal abstinence syndrome (NAS). Some degree of NAS will develop in $55 \%$ to $94 \%$ of

\footnotetext{
${ }^{1}$ ProgenyHealth, Plymouth Meeting, Pennsylvania.
}

(C) The Author(s) 2015; Published by Mary Ann Liebert, Inc. This Open Access article is distributed under the terms of the Creative Commons License Attribution-Non-Commercial Share Alike (http://creativecommons.org/licenses/by-nc-sa/4.0/). 
all neonates with in utero opioid exposure. ${ }^{7}$ Left untreated, NAS can be potentially life threatening.

A neonate with in utero drug exposure who exhibits any signs of NAS should, at minimum, receive non-pharmacologic treatment for comfort, which includes swaddling, rocking, therapeutic sucking, and minimization of sensory or environmental stimulation. To determine if an infant requires pharmacologic intervention, a scoring scale is implemented to assess the severity of NAS. The Modified Neonatal Abstinence Scoring System, often referred to as the modified Finnegan Score, is the predominant tool used in the United States. ${ }^{7}$ It provides a numeric description of the severity of the infant's symptoms and is used to decide when to initiate treatment and to determine treatment efficacy.

The mainstay of pharmacologic NAS treatment is oral opioid therapy (morphine, methadone, dilute tincture of opium [DTO]). Most physicians (94\% in the United Kingdom and $83 \%$ in the United States) use an oral opioid as the first choice of treatment, ${ }^{8}$ although there is no clear-cut optimal choice because each drug has advantages and disadvantages. ${ }^{7}$ Phenobarbital and clonidine are commonly used for polysubstance abuse, and also may be used as an adjunct to opioid therapy. ${ }^{7}$ According to a study by Sarkar, in which all 102 accredited neonatal-perinatal fellowship programs in the United States were surveyed, $63 \%$ of respondents reported using morphine or DTO, $20 \%$ reported using methadone, and $17 \%$ reported using phenobarbital as the first-line agent for managing neonatal opioid withdrawal. ${ }^{9}$

The most current American Academy of Pediatrics (AAP) guidelines published in 2012 for management of NAS recommend that each institution that cares for infants with NAS should develop a protocol that defines indications and procedures for screening delivering mothers for substance abuse. In addition, each nursery should develop and adhere to a standardized plan for the evaluation and comprehensive treatment of infants at risk for or showing signs of withdrawal. $^{7}$ However, according to the aforementioned Sarkar study, only $54.5 \%$ of all respondents report having a written NAS management policy. ${ }^{9}$ Other survey-based studies confirm the wide variability in practice patterns. ${ }^{8,10}$ The AAP also acknowledges that significant gaps in knowledge exist concerning the optimal treatment strategy (including the criteria for instituting pharmacologic therapy, the drug of first choice, and the strategy for weaning) for infants with neonatal withdrawal. ${ }^{7}$

The goal of the present retrospective data analysis is to examine all of AmeriHealth Caritas Pennsylvania's (ACP) members with NAS managed by ProgenyHealth (a provider of neonatal care management services) from 2007 through 2013. The primary outcome of the study is the average length of stay (ALOS) of this cohort. The secondary outcomes are differences in ALOS between individual hospitals and possible contributing variables, such as hospital experience, type of in utero drug exposure, and treatment regimen.

\section{Methods}

\section{Eligibility}

A search of ProgenyHealth's neonatal intensive care unit (NICU) database was performed for all ACP members born between January 1, 2007, and December 31, 2013, who fit the following eligibility criteria: $\geq 35$ weeks gestational age with a diagnosis of NAS (International Classification of Diseases, Ninth Revision code 779.5) and required pharmacologic intervention. Thirty-five weeks gestational age was chosen as a cutoff to avoid complications from prematurity affecting the length of stay. This search resulted in 152 cases, which then were subjected to the following exclusion criteria: a comorbidity significantly affecting clinical course, iatrogenic NAS, or transfer to/from another facility during NAS treatment course. This resulted in a final cohort of 139 cases from 22 hospitals.

\section{Data collection}

The following information was collected for each eligible case: admission date, discharge date, length of stay, maternal age, maternal opioid used during pregnancy, hospital where treatment occurred, primary medication used to treat NAS (including whether the treatment was completed in the hospital or as an outpatient), and any adjunctive medications used. The treatment protocol in place at each hospital was established by each individual provider group, and the historical rationale for each group's practices was not ascertainable.

\section{Data analysis}

Welch's $t$ test for independent samples was used to compare the methadone-only treatment group to the all-others treatment group, and also used to compare the methadoneexposed group to the buprenorphine-exposed group. The data are presented as mean \pm standard deviation. Regression analysis was used to look for a correlation between number of NAS cases a hospital manages and the ALOS.

\section{Results}

The ALOS of the study population was $21.1 \pm 12.4$ days. The total number of ACP NAS cases per year has been steadily increasing from 1 case in 2007 to 61 cases in 2013. In addition, the percentage of total NICU infants with a diagnosis of NAS has been increasing as well, reaching 7.3\% in 2013 (Fig. 1).

Although the recent literature suggests that neonates with in utero exposure to buprenorphine have shorter ALOS than neonates exposed to methadone, ${ }^{11}$ the current study does not reveal these findings (methadone 21.6 \pm 13.3 days, buprenorphine $22.1 \pm 10.8$ days, $P=0.43$ ). This may be because of the possibility that more infants exposed to buprenorphine may not have required pharmacologic treatment compared to their methadone-exposed counterparts, which the scope of the current study would not have captured.

However, study data show that neonates who are treated with methadone alone have a significantly shorter ALOS of $11.4 \pm 4.8$ days versus an ALOS of $25.1 \pm 12.3$ days for all other treatment regimens $(P<0.001)$ (Fig. 2). Of the 41 infants treated with methadone alone, all but 4 were discharged home to complete outpatient medication wean.

There is wide variability of ALOS within the subset of individual hospitals, and there appears to be a weak $\left(R^{2}=\right.$ $0.23)$, but significant $(P=0.019)$, correlation between number of NAS cases a hospital manages and ALOS (Fig. 3). For example, Hospital A, the institution with the greatest 


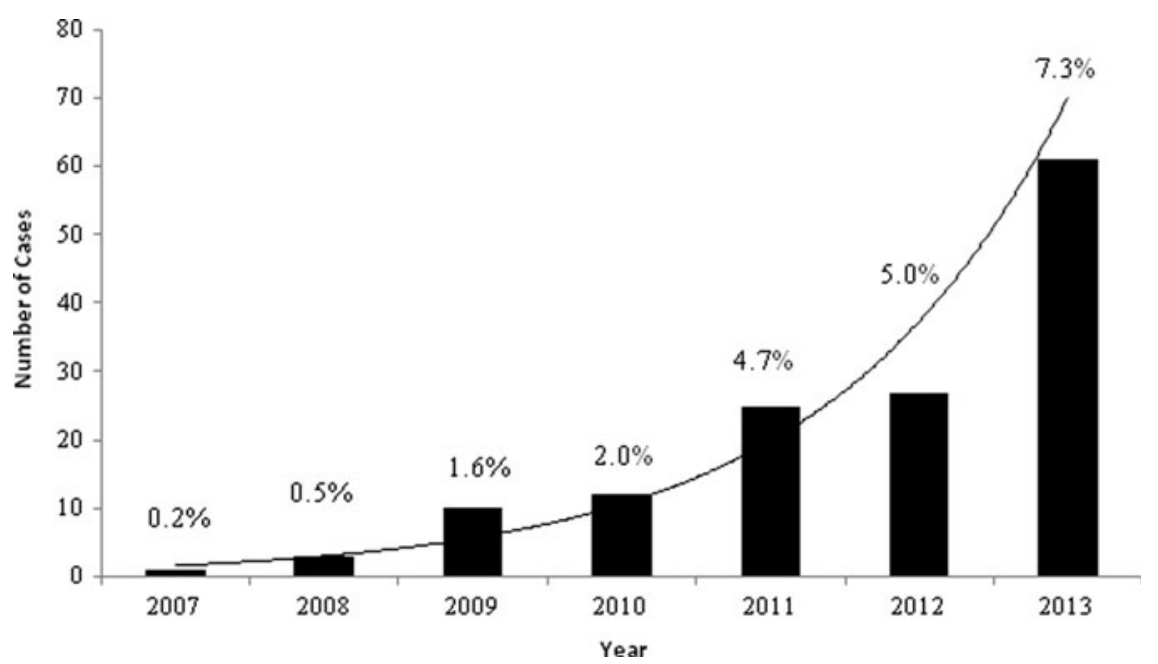

FIG. 1. Admissions for treatment of NAS and NAS as a percentage of total NICU cases. NAS, neonatal abstinence syndrome; NICU, neonatal intensive care unit.

number of ACP NAS infants during this study period, had an ALOS of between 7.3 days and 15.7 days from 2007 until the first half of 2013, when all of the NAS babies were treated with a combined inpatient/outpatient methadone regimen. However, in the second half of 2013, all of the NAS babies at Hospital A were treated with morphine (with or without adjunctive medication) entirely as an inpatient, resulting in an ALOS of 37.5 days (Fig. 4).

Of the 139 cases in the study cohort, there were 12 discrete patient readmissions in the first year of life (Table 1). Ten of the 12 readmissions were not directly related to the initial NAS diagnosis. One readmission was for suspected seizure in a baby who was previously treated with morphine while in the NICU. The infant received a sepsis workup after readmission, did not exhibit any other withdrawal symptoms, and did not receive any subsequent opioid therapy. One readmission was for an infant discharged from the NICU to complete outpatient methadone treatment, who required readmission for worsening NAS symptoms. The patient had missed 2 consecutive doses of methadone secondary to maternal inability to procure additional methadone doses. This infant completed his NAS treatment as an inpatient with morphine with a 28-day length of stay.

\section{Discussion}

The results of this retrospective study confirm that ACP admissions for NAS have been increasing steadily, mirroring the national trend. This is of particular importance for ACP because the mean hospital charge for infants diagnosed with NAS is $\$ 53,400$, and $77.6 \%$ of charges for NAS are attributed to state Medicaid programs. ${ }^{6}$ The overall ALOS of 21.1 days for the current study population compares favorably to a consensus duration of approximately 30 days

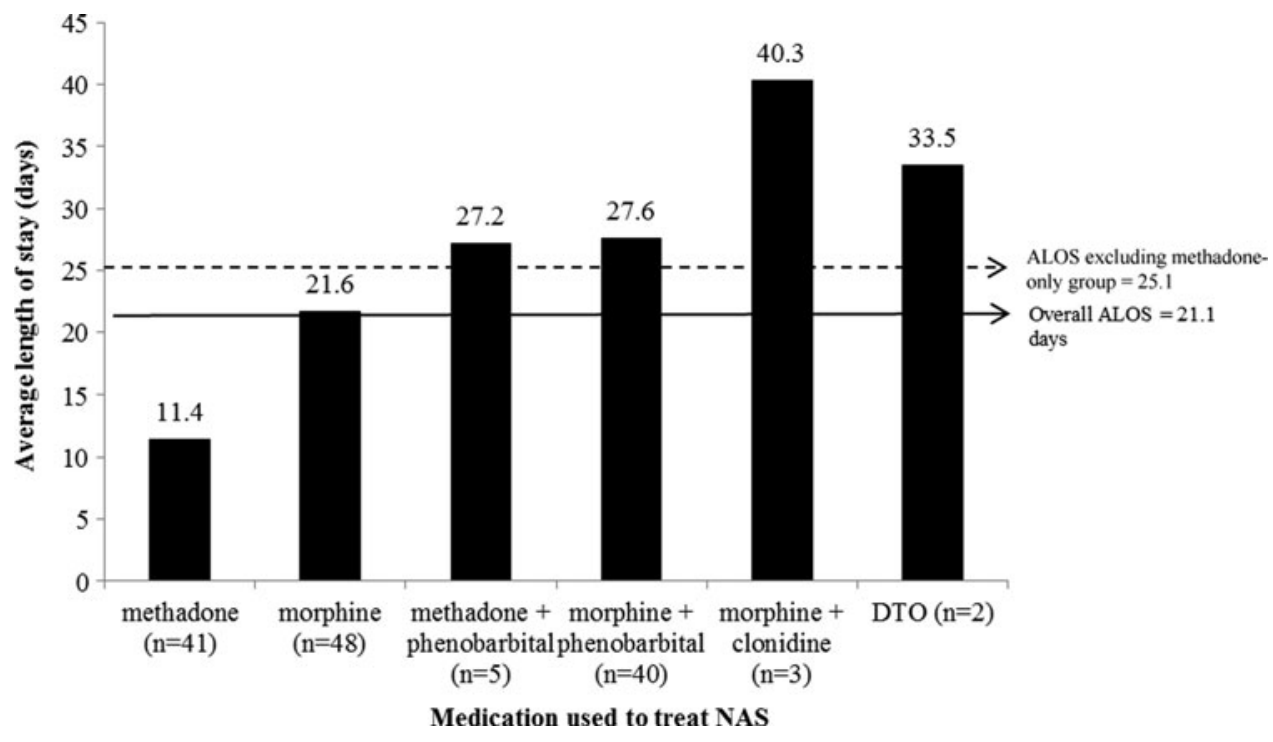

FIG. 2. Average length of stay secondary to NAS treatment. ALOS, average length of stay; DTO, dilute tincture of opium; NAS, neonatal abstinence syndrome. 


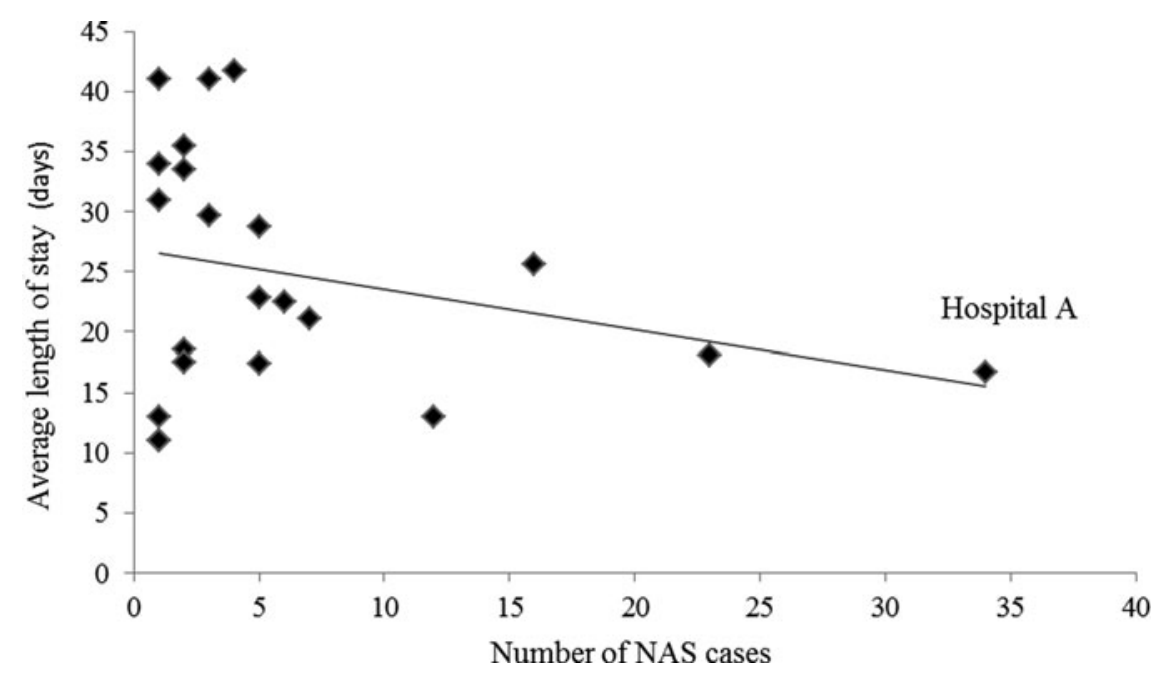

FIG. 3. Average length of stay as a function of hospital experience. NAS, neonatal abstinence syndrome.

reported by Kraft and Seligman. ${ }^{12,13}$ When ALOS was evaluated further by treatment regimen, it can be seen that infants who began their treatment in the hospital with methadone and were discharged home to complete outpatient treatment had an ALOS of 11.4 days versus 25.1 days for infants who completed their entire treatment in the hospital. This $55 \%$ reduction in ALOS translates to approximately $\$ 29,150$ in savings for each case of NAS, or $\$ 396$ million per year nationally based on an estimated 13,600 cases of NAS in $2009 .^{6}$

This finding is consistent with a recent retrospective study by Backes et al, ${ }^{14}$ which found a $48 \%$ decrease in length of stay for infants treated in a combined inpatient/outpatient treatment group (13 days) as compared to the inpatient-only treatment group (25 days), although the combined inpatient/ outpatient group required an overall longer duration of treatment. Additionally, in infants completing their methadone treatment at home, Backes et al found no increased risk of short-term adverse outcomes, emergency room visits, hospital readmission, or need for inpatient treatment for NAS.
The findings of the current study also are in line with a recent large retrospective cohort study by Patrick and colleagues, ${ }^{15}$ which looked at variation in NAS treatment at 14 children's hospitals and found that wide variations in treatment and outcomes exist among the hospitals. In addition, the authors report first-line treatment with methadone resulted in shorter length of stay when compared with morphine, although, unlike the current study, the reduction was independent of discharge patterns.

The experience reported from Hospital A is similar to an experience published in 2013 by Napolitano and colleagues, ${ }^{16}$ in which they report on a combined inpatient/ outpatient methadone program from April 2008 through July 2010 with an ALOS of 12 days. However, because of concerns raised by community pediatricians regarding outpatient NAS management, they switched to an all inpatient treatment program with morphine with a resultant increase in ALOS to 29 days. Since then, with continued intensive refinement, they have been able to decrease their ALOS to 20 days through the formation of an NAS Committee that

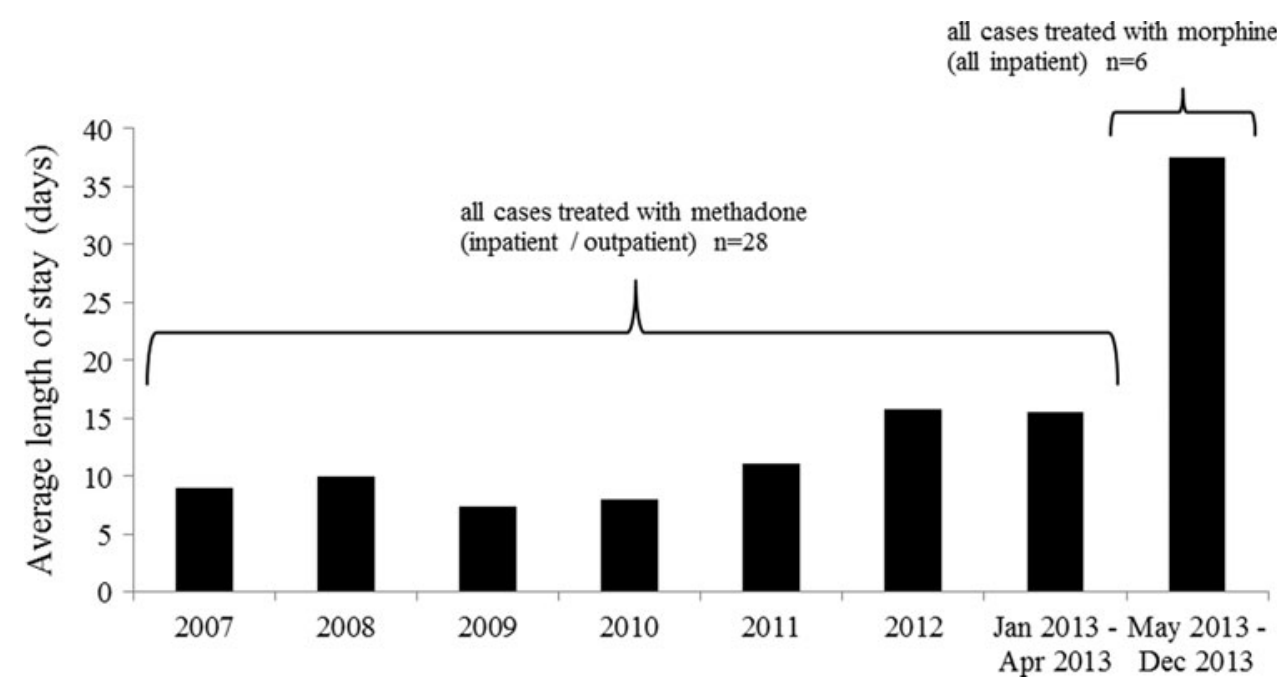

FIG. 4. Average length of stay at Hospital A from 2007-2013. 
Table 1. Readmissions in the First Year of Life.

\begin{tabular}{|c|c|c|}
\hline Readmission & Age & Related to NAS? \\
\hline Right arm fracture & 5 months & No \\
\hline Asthma exacerbation & 8 months & No \\
\hline Groin abscess & 4 months & No \\
\hline Pyloric stenosis & 2 months & No \\
\hline Tracheomalacia & 1 month & No \\
\hline Rotavirus infection & 2 weeks & No \\
\hline Bronchiolitis & 8 months & No \\
\hline Pyloric stenosis & 1 month & No \\
\hline Pneumonia & 6 months & No \\
\hline RSV bronchiolitis & 9 months & No \\
\hline Possible seizure & $\begin{array}{l}5 \text { days after } \\
\text { discharge }\end{array}$ & $\begin{array}{l}\text { Likely not. Infant was } \\
\text { originally treated } \\
\text { with morphine as } \\
\text { inpatient. For } \\
\text { readmission, infant } \\
\text { was discharged } \\
\text { without any NAS } \\
\text { treatment after } \\
\text { negative sepsis } \\
\text { evaluation }\end{array}$ \\
\hline NAS & 1 month & $\begin{array}{l}\text { Yes; mother was } \\
\text { unable to fill } \\
\text { methadone } \\
\text { prescription as } \\
\text { outpatient and } \\
\text { missed doses }\end{array}$ \\
\hline
\end{tabular}

NAS, neonatal abstinence syndrome; RSV, respiratory syncytial virus.

meets quarterly to refine the treatment guideline and to investigate barriers to discharge. One program involved cooperation between the county Department of Children and Family Services in-house representative and hospital case management to assess maternal capability and provide early intervention/education to prepare for discharge, which remained available to the mothers after discharge. In the case of Hospital A, the decision to change from a combined inpatient/outpatient treatment regimen with methadone to an entirely inpatient regimen with morphine was related to a change in treatment preference of a new provider group at the hospital. Unlike Hospital A, the treatment practices (with regard to first-line medication) at the other hospitals did not vary during the analysis time frame. Thus, the 6 cases of NAS at Hospital A treated with morphine as an inpatient in the second half of 2013 likely had minimal effect on the overall length of stay results.

In the data analysis of the current study, there was 1 treatment failure requiring readmission for exacerbation of NAS symptoms as a result of missed methadone doses from a caregiver unable to obtain additional outpatient methadone.

Limitations of this study include its retrospective nature and small sample size. An additional limitation is procurement of cases from a database, which relies on accuracy of data entry. A third limitation is the lack of access to data sets containing other potential confounding variables influencing length of stay, such as family/social issues, staff training, and breastfeeding rates. Also, this evaluation of hospital experience is based on the number of cases from a single health plan; it could be that certain hospitals see disproportionately more NAS cases from other health plans.
Arguments against outpatient treatment focus on the need for inpatient monitoring as these medications can cause respiratory depression, diversion of medication, risk of overdosage or underdosage leading to morbidity, infant abuse, and inability to secure a compounding pharmacy for methadone. ${ }^{5,16,17}$ However, if the infant is stabilized and weaned to a low dose, appropriate caregivers are carefully selected with comprehensive social services engaged, and a dedicated outpatient program is in place with educated, experienced providers and unencumbered access to medication, this can be a safe and cost-effective management strategy. Other advantages include decreasing exposure to hospital-associated morbidity, and facilitating establishment of the mother-baby dyad including breast-feeding, which has been shown to decrease the severity of NAS symptoms ${ }^{18,19,20}$ and is supported by the $\mathrm{AAP}^{21}$ and the American College of Obstetricians and Gynecologists. ${ }^{22}$ The risk of adverse effects can be further mitigated by discharge with a home apnea monitor, a strategy many programs currently employ. Strong clinical and social supports are a keystone to outpatient management. Program success can be bolstered by persistent case management services and a centralized location with physicians, nurses, and social workers present, along with a pharmacy that can dispense methadone in prefilled doses. The authors believe that this study provides further evidence that a combined inpatient/outpatient NAS treatment regimen with methadone can significantly decrease length of stay and associated health care expenditures without increased adverse outcomes. Development of successful combined inpatient/outpatient management programs for NAS warrants further consideration.

\section{Author Disclosure Statement}

Drs. Lee, Hulman, Musci, and Stang declared the following potential conflicts of interest with respect to the research, authorship, and/or publication of this article: All authors are employed by ProgenyHealth, which funded this study.

\section{Acknowledgment}

The authors would like to thank Marge Angello, RN, Market President of AmeriHealth Caritas Pennsylvania, for her support of ProgenyHealth.

\section{References}

1. Manchikanti L, Singh A. Therapeutic opioids: a ten-year perspective on the complexities and complications of the escalating use, abuse, and nonmedical use of opioids. Pain Physician. 2008;11(2 suppl):S63-S88.

2. DeNoon DJ. The 10 most prescribed drugs. http://www .webmd.com/news/20110420/the-10-most-prescribed-drugs. Accessed January 24, 2014.

3. Manchikanti L, Fellows B, Ailinani H, Pampati V. Therapeutic use, abuse, and nonmedical use of opioids: a tenyear perspective. Pain Physician. 2010;13:401-435.

4. SAMHSA (Substance Abuse, and Mental Health Services Administration). Results from the 2010 national survey on drug use and health: summary of national findings. Rockville, MD: US Department of Health and Human Services;2011. 
5. Grim K, Harrison TE, Wilder RT. Management of neonatal abstinence syndrome from opioids. Clin Perinatol. 2013; 40:509-524.

6. Patrick SW, Schumacher RE, Benneyworth BD, Krans EE, McAllister JM, Davis MM. Neonatal abstinence syndrome and associated health care expenditures United States, 2000-2009. JAMA. 2012;307:1934-1940.

7. Hudak ML, Tan RC. Neonatal drug withdrawal. Pediatrics. 2012;129:e540-e560.

8. O'Grady MJ, Hopewell J, White MJ. Management of neonatal abstinence syndrome: a national survey and review of practice. Arch Dis Child Fetal Neonatal Ed. 2009;94:F249-F252.

9. Sarkar S, Donn SM. Management of neonatal abstinence syndrome in neonatal intensive care units: a national survey. J Perinatol. 2006;26:15-17.

10. Crocetti MT, Amin DD, Jansson LM. Variability in the evaluation and management of opiate-exposed newborns in Maryland. Clin Pediatr (Phila). 2007;46:632-635.

11. Jones HE, Kaltenbach K, Heil SH, et al. Neonatal abstinence syndrome after methadone or buprenorphine exposure. N Engl J Med. 2010;363:2320-2331.

12. Kraft WK, Gibson E, Dysart K, et al. Sublingual buprenorphine for treatment of the neonatal abstinence syndrome: a randomized trial. Pediatrics. 2008;122: e601-e607.

13. Seligman NS, Salva N, Hayes EJ, Dysart KC, Pequignot EC, Baxter JK. Predicting length of treatment for neonatal abstinence syndrome in methadone-exposed neonates. Am J Obstet Gynecol. 2008;199:396e1-396e7.

14. Backes $\mathrm{CH}$, Backes $\mathrm{CR}$, Gardner $\mathrm{D}$, Nankervic CA, Giannone PJ, Cordero L. Neonatal abstinence syndrome (NAS): transitioning methadone treated patients from an inpatient to outpatient setting. J Perinatol. 2012;32:425430.

15. Patrick SW, Kaplan HC, Passarella M, Davis MM, Lorch SA. Variation in treatment of neonatal abstinence syndrome is US children's hospitals, 2004-2011. J Perinatol. 2014; 34:867-872.

16. Napolitano A, Theophilopoulos D, Seng SK, Calhoun DA. Pharmacologic management of neonatal abstinence syndrome in a community hospital. Clin Obstet Gynecol. 2013; 56:193-201.

17. Jansson LM, Velez M, Harrow C. The opioid exposed newborn: assessment and pharmacologic management. J Opioid Manag. 2009;5:47-55.

18. Abdel-Latif ME, Pinner J, Clews S, Cooke F, Lui K, Oei J. Effects of breast milk on the severity and outcome of neonatal abstinence syndrome among infants of drugdependent mothers. Pediatrics. 2006;117:e1163-e1169.

19. Pritham UA. Breastfeeding promotion for management of neonatal abstinence syndrome. J Obstet Gynecol Neonatal Nurs. 2013;42:517-526.

20. Dryden C, Young D, Hepburn M, Mactier H. Maternal methadone use in pregnancy: factors associated with the development of neonatal abstinence syndrome and implications for healthcare resources. BJOG. 2009;116:665-671.

21. American Academy of Pediatrics. Breastfeeding and the use of human milk. Pediatrics. 2012;129:e827-e841.

22. ACOG Committee on Health Care for Underserved Women; American Society of Addiction Medicine. ACOG Committee Opinion No. 524: Opioid abuse, dependence, and addiction in pregnancy. Obstet Gynecol. 2012;119: 1070-1076.
Address correspondence to: Dr. Jerry Lee ProgenyHealth 450 Plymouth Road, Suite 200 Plymouth Meeting, PA, 19462

E-mail: jlee@progenyhealth.com 